\title{
Optical Polarization Errors in Sagnac Fiber Optic Current Sensor
}

\author{
Wang Lihui $^{1,3}$, Sun Jian ${ }^{2}$, Ji Jianfei ${ }^{2}$ and Xu Shengbao ${ }^{1}$ \\ ${ }^{1}$ Key Laboratory of micro-inertial instrument and advanced navigation technology, Ministry of education, School of instrument \\ science and engineering, Southeast University, Nanjing 210096, China \\ ${ }^{2}$ Jiangsu Electrical Power Company Research Institute, Nanjing, 211103, China \\ ${ }^{3}$ State Key Laboratory of Transient Optics and Technology, Xi an Institute of Optics and Precision Mechanics, Chinese Academy \\ of Sciences, Xi an 710119, China \\ wlhseu@163.com
}

\begin{abstract}
Sagnac fiber optic current sensor(S-FOCS) is a kind of precision instrument based on optical polarization and optical interference theory. There are several kinds of error characteristics from optical components in S-FOCS, including polarizer, integrated optical chip, quarter-wave retarder, sensing head and so on. With these errors, linearly polarized light wave in PM fiber and circularly polarized light wave in sensing fiber become elliptically polarized light wave, then, nonreciprocal phase shift induced by magnetic field of the current is interrupted by wrong polarization state. Focused on optical polarization error in S-FOCS, we analyze the characteristics of polarization errors associated with optical components in S-FOCS theoretically, and we demonstrate the methods to build optical polarization error models by using Poincare sphere and Jones matrix respectively. Then, based on model of polarization state in S-FOCS, we carry out simulation of polarization error characteristics in SFOCS, and theoretically investigate the influence of several main error factors on optical polarization characteristics, including polarizer, phase delay in quarter-wave retarder, splice angular between quarter-wave retarder and PM fiber.

Index Terms - Fiber optic current sensor, Fiber optic gyroscope, Optical polarization state, Phase retardation, Modeling.
\end{abstract}

\section{Introduction}

Sagnac fiber optic current sensors (S-FOCS) have a number of inherent advantages over conventional current sensing techniques[1,2], which becomes very attractive for metering, control, and protection in high-voltage substations, especially in harsh environments, including broad dynamic range, high measuring accuracy, excellent insulating performance, and so on. In S-FOCS, the nonreciprocal phase shift is accumulated by circularly polarized light waves traveling in opposite polarization directions with respect to the magnetic field produced by the current to be measured. To restrict optic amplitude polarization error and optic intensity polarization error in S-FOCS, it is, therefore, necessary to produce and maintain high-quality states of circular polarization within the sensing fiber[3], and high-quality states of linear polarization within the polarization maintaining (PM) fiber. In practice, several key components in optic loop show error characteristics, including polarizer, quarter-wave retarder and sensing head, which induces linearly polarized light wave in PM fiber and circularly polarized light wave in sensing fiber to be elliptically polarized light wave, then nonreciprocal phase shift induced by magnetic field of the current is interrupted by wrong polarization state.

Focusing on optical polarization error in Sagnac fiber optic current sensor, in this paper, propagation process of light wave is analyzed, polarization model is demonstrated, and methods to evaluate the influence of polarization error to $\mathrm{S}$ FOCS's performance are proposed. First, we describe polarization characteristic in S-FOCS by using Poincare sphere method, and build model of polarization state by using Jones matrix. Then, based on model of polarization state in SFOCS, we theoretically investigate the influence of several main error factors on optical polarization characteristics, including polarizer, phase delay in quarter-wave retarder, splice angular between quarter-wave retarder and PM fiber. Finally, we quantify nonreciprocal phase shift to be detected in S-FOCS with optical polarization error.

\section{Description of Optical Polarization State in S-FOCS}

The structure of interferometric Sagnac fiber optic current sensor is shown in Fig. 1, the light wave propagation process in S-FOCS can be described as follows. Light from source is directed through a single-mode fiber coupler to a fiber polarizer. The polarized light is equally split into two beams, and converted to circularly polarized light waves by a quarterwave retarder just prior to the sensing region. Two beams light travel in opposite directions with respect to the magnetic field, and return to linear polarization states on passing through the quarter-wave retarder on the return trip, finally, they back together and interfered in polarizer and coupled to the detector. A phase shift caused by the magnetic field is produced between the two circularly polarized polarization modes in the sensing region, which is two times the single-pass Faraday rotation[4].

Mutual conversion between linear polarization state and circular polarization state is an important issue, and the conversion process can be analyzed with Jones matrix as follows. Non-linear-polarized light wave emitted from SLD with Jones matrix [Px Py]T travels through polarizer, and light wave in y axis is filtered, therefore, [Px Py]T becomes linear polarized light wave [Px 0] T. As described above, light wave [Px 0] T experiences $45^{\circ}$ splice and quarter-wave retarder. 


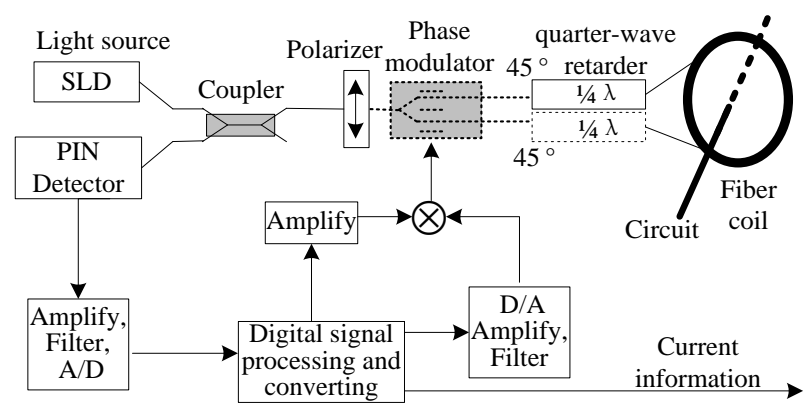

Fig. 1 Structure of Sagnac fiber optic current sensor

With linearly polarized light wave Px from polarizer, matrix of light wave component of Px passing through angular $\gamma$ and quarter-wave retarder is given by

$$
[M]_{P x}=[M]_{\gamma, \lambda / 4}\left[\begin{array}{c}
0 \\
P_{x}
\end{array}\right]=\left[\begin{array}{l}
{[\sin \gamma \cos \gamma(1-j)] P_{x}} \\
{\left[\sin ^{2} \gamma+j \cos ^{2} \gamma\right] P_{x}}
\end{array}\right]
$$

where $\gamma$ is angular between axes of PM fiber and quarter-wave retarder, $[\mathrm{M}]_{\gamma, \lambda 4}$ is Jones matrix of quarter-wave retarder. Eq (2) shows that $P_{x}$ changes to circularly polarized light wave.

$$
[M]_{\gamma, \lambda / 4}=\left[\begin{array}{cc}
\cos ^{2} \gamma+j \sin ^{2} \gamma & \sin \gamma \cos \gamma(1-j) \\
\sin \gamma \cos \gamma(1-j) & \sin ^{2} \gamma+j \cos ^{2} \gamma
\end{array}\right]
$$

\section{III . Modeling of optical polarization characteristics in S- FOCS}

To meet the index requirement of FOCS accuracy within $\pm 0.2 \%$, it is critical to restrain polarization errors[5], which result from spurious interference of light traveling around the optical loop in the wrong polarization state. There are several kinds of polarization state in S-FOCS, which makes polarization analysis of S-FOCS complex, as polarization state may change anywhere within PM fiber and single mode sensing coil, and some light couples to the wrong polarization state by means of polarization cross-coupling, depending on optical components' performance and fiber's birefringence induced by internally or externally stresses, such as inherent imperfections, temperature, vibration, and so on. Polarization state changes fast enough to introduce a phase difference between counter propagating waves, such as time varying nonreciprocal. These errors appear as a nonreciprocal phase shift indistinguishable from that induced by current, and influence the bias and scale factor stability of S-FOCS[1, 6, 7]. Jones matrix provide a mathematically rigorous method of tracking polarization, however, they often lead to a less intuitive description. Despite the mathematical complexity of some phenomena observed in S-FOCS, the Poincare sphere provides elegant and simple geometrical interpretations. Focused on polarization characteristics in S-FOCS, this section describes polarization evolving process with Poincare sphere and Jones matrix, then, builds mathematical model of polarization state inS-FOCS. Jones matrix and Poincare sphere are combined, and they offer both a mathematical representation and an intuitive interpretation of nonreciprocal polarization effects in S-FOCS.

Polarization state of light wave can be expressed clearly on Poincare sphere. By using retardation $\gamma$ and rotation $\delta$, we can convert any polarization state into a new polarization state[1]. Such a vectorial description of polarization represents three out of four elements of Stokes vector. Polarization state vector $\vec{P}$ can be given by

$$
\vec{P}\left(\overrightarrow{P_{0}}, \overrightarrow{P_{45}}, \overrightarrow{P_{c}}\right)=(\cos 2 \gamma, \sin 2 \gamma \cos \delta, \sin 2 \gamma \sin \delta)
$$

$\vec{P}_{0}$ represent $0^{\circ}$ linear polarization state with $\gamma=0^{\circ}$ and $\delta=0^{\circ}$, $\overrightarrow{P_{45}}$ represent $45^{\circ}$ linear polarization state with $\gamma=45^{\circ}$ and $\delta=0^{\circ}$, $\overrightarrow{P_{c}}$ represent circular polarization state with $\gamma=45^{\circ}$ and $\delta=90^{\circ}$.

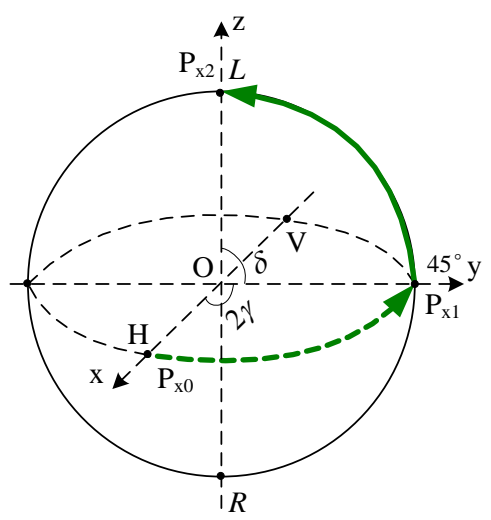

Fig. 2 Polarization from linear to circular

Fig. 2 shows optical polarization evolution from linear polarization state to circular polarization state. Horizontal linearly polarized light $P_{x 0}$ in PM fiber rotates angle of $2 \gamma$ to $P_{x 1}$ along the equator, then $P_{x 1}$ rotates phase retardation angle of $\delta$ to $P_{x 2}$ along longitude. After transmission in sensor head, the two waves pass through quarter-wave retarder, optical polarization evolutes from circular polarization state to linear polarization state, and the process can be described in Fig.3.

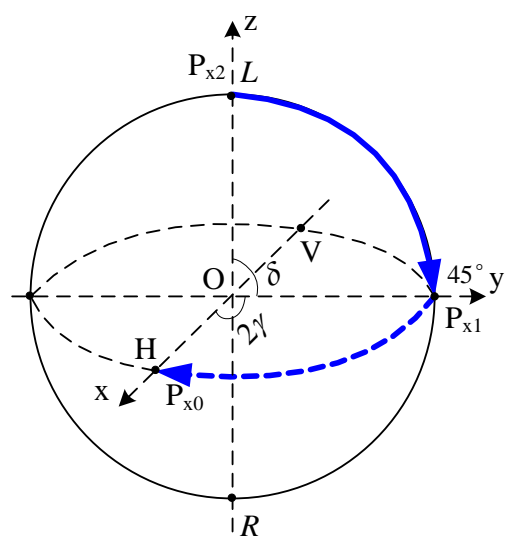

Fig. 3 Polarization from circular to linear 


\section{IV . Polarization error characteristics in S-FOCS}

In practice, polarization performance of light-wave in SFOCS is susceptible to several factors, such as inherent defects of optical components, temperature stability, vibration, equipment aging, and current value deviates from the theory value. Polarization error sources in S-FOCS include linearly polarized error and circularly polarized error. Linearly polarized error source includes polarization degree error of polarized light-wave traveling from polarizer, integrated optical chip splitting ratio error, $45^{\circ}$ splice angular alignment error between PM fiber and retarder, and sensing fiber linear birefringence error. Circularly polarized error source includes $90^{\circ}$ phase shift error in retarder.

A. Polarization Degree Error of Polarized Light - wave Traveling from Polarizer

Polarizer lies in common point with light-wave's entering and exiting, and has the function to filter polarized light-wave undesired, which ensures reciprocity of light-wave in S-FOCS. Polarizer's performance influence measurement accuracy, imperfect polarizer will induce polarization state randomly coupling, and express intensity-type polarization error in SFOCS $[1,3,4]$.

The output intensity resulting from the interference of two returning linear optic waves is given by

$$
\begin{aligned}
& I=I_{0} \cdot[1+\cos \varphi(I)] \\
& =I_{0} \cdot\left[\left(1+\varsigma^{2}\right)^{2}+\left(1-\varsigma^{2}\right)^{2} \cos \left(\varphi_{\bmod }(t)+2 N V I\right)\right]
\end{aligned}
$$

where $S$ is error factor of polarizer, $M_{p o}$ is Jones matrix of polarizer. Eq. (4) shows that the output intensity of light-wave interference signal is disturbed by polarizer's error factor $\varsigma$, then, the capacity of minimum current resolution in S-FOCS is decreased. Nonreciprocal phase shift induced by Faraday magnetic field are interested, then, light-wave interference signal intensity $\left(1-\varsigma^{2}\right)^{2} I_{0}$ has no effect on phase to be measured by using closed loop control technology, therefore, imperfect polarizer doesn't affect measurement accuracy, phase to be measured of $\varphi_{s}(t)$ still meets the relationship of $\varphi_{s}(t)=-2 N V \cdot I$, on condition of meeting minimum current resolution requirement.

\section{B. Integrated Optical Chip Splitting Ratio Error}

Integrated optical chip (IOC) in S-FOCS outputs two beams of light wave, named Ix and Iy, as affected by process factors, light-wave intensity distributes unequally. The output intensity resulting from the interference of two returning linear optic waves is given by

$$
\begin{aligned}
& I=2 \sqrt{I_{x} I_{y}}\left\{1+\cos \left[\varphi(I)+\varphi_{\text {mod }}(t)\right]\right\} \\
& =\sqrt{2} I_{0} \sqrt{\cos (2 \alpha)}\left\{1+\cos \left[\varphi(I)+\varphi_{\text {mod }}(t)\right]\right\}
\end{aligned}
$$

Phase to be measured of $\varphi(I)$ is not disturbed by integrated optical chip splitting ratio error, in spite of light-wave intensity is influenced.

\section{Axial Splice Angular Alignment Error between IOC and Retarder}

Jones matrix of quarter-wave retarder with splice angular of $\gamma$ can be obtained by Eq.(2). The output intensity resulting from the interference of two returning linear optic waves is given by

$$
I=I_{0} \cdot\left\{\begin{array}{l}
1-(1-\sin 2 \gamma) \cos \varphi_{\text {mod }}(t) \\
+\sin 2 \gamma \cos \left[\varphi(I)+\varphi_{\text {mod }}(t)\right]
\end{array}\right\}
$$

To eliminate stationary bias in signal processing unit, the subtraction is designed between the accumulation of the signal intensity in the positive and negative offset square wave, and we can obtain

$$
\varphi_{s}(t)=\arctan \left[-\frac{\sin 2 \gamma \sin \varphi(I)}{\sin 2 \gamma-1+\sin 2 \gamma \cos \varphi(I)}\right]
$$

It can be seen from Eq.(7) that the relationship between the recovered phase shift $\varphi_{s}(t)$ and the Faraday phase shift $\varphi(I)$ of S-FOCS with imperfect $45^{\circ}$ splice angular between PM fiber lead and quarter-wave Retarder is nonlinear, as the relationship between $\varphi(I)$ and I is linear, therefore, the scale factor of S-FOCS with imperfect $45^{\circ}$ splice angular is nonlinear.

The relationship between scale factor and splice angular is shown in Fig. 4. It can be seen that the recovered phase shift $\varphi_{s}(t)$ in S-FOCS with imperfect $45^{\circ}$ splice angular between

PM fiber lead and quarter-wave retarder is nonlinear, as the relationship between $\varphi(I)$ and I is linear, therefore, the scale factor of S-FOCS with imperfect $45^{\circ}$ splice angular is nonlinear.

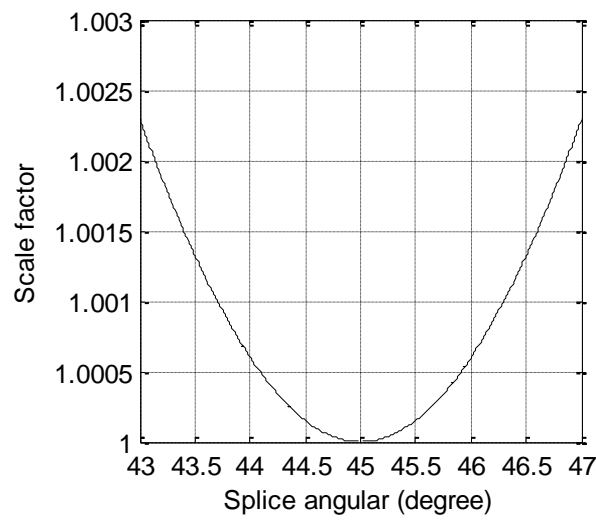

Fig. 4 Scale factor with splice angular error 


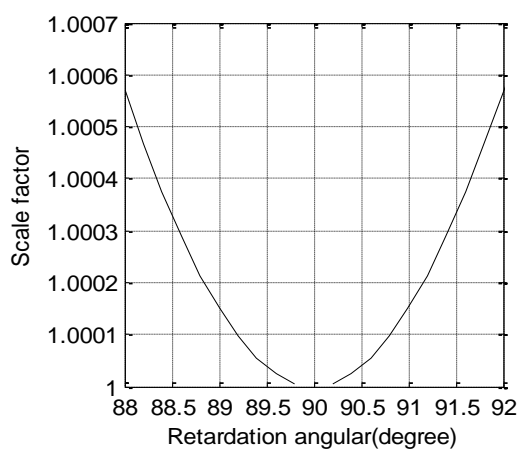

Fig. 5 Scale factor with phase retardation error

\section{Conclusion}

Polarization error characteristics affect the accuracy and stability of the Sagnac fiber optic current sensor, then, we have carried out a theoretical investigation on the polarization error characteristics against the variations of the quality of polarizer, phase delay in quarter-wave retarder, $45^{\circ}$ splice angular between quarter-wave retarder and PM fiber in S-FOCS. Imperfect polarizer in S-FOCS induces polarization degree error, however, polarized light-wave passing through the polarizer doesn't affect measurement accuracy, and phase to be measured in S-FOCS still meets the relationship of $2 \mathrm{VN}$, on condition of meeting minimum current resolution requirement. Intensity of two light-wave in fiber loop with clockwise and counterclockwise propagation direction distributes unequally, when splitting ratio of integrated optical chip in S-FOCS deviates 50:50, however, the splitting ratio error has no effect on S-FOCS's measurement accuracy. However, the scale factor of Sagnac fiber interferometer current sensor deviates from the value $2 \mathrm{VN}$ when either the quarter-wave retarder is imperfect, or splice angular between quarter-wave retarder and $\mathrm{PM}$ fiber not equal $45^{\circ}$. In addition, linear birefringence in the fiber sensing lead is still major polarization error factors to consider.

\section{VI . Acknowledgment}

The project is supported by the following funds:
Fundamental Research Funds for the Central Universities, Research Project of Jiangsu Electrical Power Company(2013), National Natural Science Foundation of China (61203192), Natural Science Foundation of Jiangsu Province (BK2012326), Open Research Fund of State Key Laboratory of Transient Optics and Photonics, Chinese Academy of Sciences(SKLST201108), Foundation of Key Laboratory of Micro-Inertial Instrument and Advanced Navigation Technology, Ministry of Education.

\section{References}

[1] M. King, B. Zhu, and S. Tang, "Optimal path planning," Mobile Robots, vol. 8 , no. 2 , pp. 5 .

[2] L.Wang, X, X. Liu, et al, "Modeling and simulation of polarization errors in reflective fiber optic current sensor," Optical Engineering, vol. 50, no. 7, pp.1-7.

[3] K. Bohnert, P. Gabus, J. Nehring, et al., "Temperature and Vibration Insensitive Fiber-Optic Current sensor," Journal of Lightwave Technology, vol. 20, no. 2, pp. 267-276.

[4] S. Short, A. Tselikov, J. de Arruda, et al, "Imperfect Quarter-Waveplate Compensation in Sagnac Interferometer-Type Current Sensors," Journal of Lightwave Technology, vol. 16, no. 7, pp. 1212-1219.

[5] X. Wang, C. Zhang, C. Zhang, et al, "Study of Polarization Errors of All Fiber Optical Current Transformers," Acta Pho Sin, vol. 36, no. 2, pp. 320-323.

[6] K. Bohnert, P. Gabus, J. Kostovic, et al, "Optical fiber sensors for the electric power industry," Optics and Lasers in Engineering, vol. 43, no. 2, pp. 511-526.

[7] M. Oh, W. Chu, K. Kim, et al, "Polymer waveguide integrated-optic current sensors," Optics Express, vol. 19, no.10, pp. 9392-9400.

[8] M. Belal, Z. Song, Y. Jung, G. Brambilla, et al, "Optical fiber microwire current sensor," Optics Letters, vol. 35, no.18, pp. 3045-3047.

[9] M. Belal, Z. Song, Y. Jung, et al, "An interferometric current sensor based on optical fiber micro wires," Optics Express, vol. 18, no. 19, pp. 19951-19956.

[10]A. Rose, J. Blake, "Optical Current sensors for Electric Power Grid Modernization," Optics and Photonics for Advanced Energy Technology (Energy), Cambridge, Massachusetts, June 24, 2009.

[11]K. Bohnert, P. Gabus, J. Nehring, "Fiber-Optic Current sensor for Electrowinning of Metals," Journal of Lightwave Technology, vol. 25, no.11, pp. 3602-3609.

[12]C. Kang, W. Lv, H. Ouyang, et al, "The Separate Detection of Linear Birefringence and Faraday Effect in Optical Current Transformer," Acta Optica Sinica, vol. 28, no. 1, pp. 163-168. 